\title{
Adverse effects of lipocryolysis: Analysis of 28 cases
}

\author{
Rafaela Vidal $^{1}$, Laura Segura ${ }^{2}$, Paulo Vergara ${ }^{3}$, Hernán Pinto ${ }^{4}$ \\ ${ }^{1}$ IM Clinic, Sant Cugat del Vallès, Spain \\ ${ }^{2}$ Policlínico Salud 4, La Coruña, Spain \\ ${ }^{3}$ IMEBA, Palma, Spain \\ ${ }^{4}$ Instituto de Investigaciones para las Especialidades Estéticas y del Envejecimiento (i2e3), Barcelona, Spain
}

\section{Email address:}

rafaelavidal10@hotmail.com (R. Vidal),doctora_segura@hotmail.com (L.Segura), paiovergara@ hotmail.com (P. Vergara), hpinto@i2e3.com (H. Pinto)

\section{To cite this article:}

Rafaela Vidal, Laura Segura, Paulo Vergara, Hernán Pinto. Adverse Effects of Lipocryolysis: Analysis of 28 Cases. Journal of Surgery. Special Issue: Breakthroughs in Aesthetic Medicine. Vol. 3, No. 1-1, 2015, pp. 6-7. doi: 10.11648/j.js.s.2015030101.12

\begin{abstract}
Introduction: Lipocryolysis is an effective and safe technique for the treatment of localized adiposities. However, there is very little evidence regarding its adverse effects. Materias and Methods: Retrospective analysis of 28 clinical records. Results: The adverse effects of lipocryolysis are mild to moderate, and reversible. Discussion: Specific studies need to be carried out in order to survey adverse effects in a large number of subjects, including medium/long-term follow-up.
\end{abstract}

Keywords: Lipocryolysis, Adverse Effects

\section{Introduction}

According to today's beauty standards, body image is negatively affected by unwanted localized subcutaneous fat. Fatty tissue can be surgically removed by conventional abdominoplasty [1] or by liposuction. Other, non-invasive therapies, such as laser [2], radiofrequency [3] and ultrasound [4], are also available to try to destroy subcutaneous fatty tissue. One of such non-invasive techniques is lipocryolysis [5], which combines tissue heat extraction with vacuum for the selective damaging of adipocytes and the reduction of subcutaneous fat $[6,7]$, with no damage to any other tissues. This technique has become established as a safe alternative with proven effectiveness [8] and minimal complications.

Coleman et al [9] assessed biopsies from patients with post-session hyposthesia and shown the absence of structural damage or any changes in peripheral nerve fibers, as well as process reversibility in $100 \%$ of cases. Other studies have applied lipocryolysis on flanks, observing a mild decrease in HDL (not below $4 \mathrm{mg} / \mathrm{dl}$ ) concomitant with a discrete increase in serum triglycerides (not over $11 \mathrm{mg} / \mathrm{dl}$ ), both values normalizing starting at 12 weeks post-treatment [10, 11]. Lastly, there have been two reports of paradoxical adipose hyperplasia (PAH), an adverse effect which had never been reported before associated with a lipocryolysis session, indicating that its frequency is extremely low [12].

To date, only one study has explored the adverse effects of lipocryolysis in general, concluding that complications are usually mild and associated with the trauma caused by suction [13].

The great interest in this technique, the current lack of evidence for the development of new and improved protocols, and the lack of information regarding the determinants of its adverse effects have motivated this paper, whose purpose has been to assess the frequency of the adverse effects of lipocryolysis in the treatment of localized adiposities.

\section{Material and Methods}

A retrospective analysis of the medical record of the patients who had undergone a session of lipocryolysis on the abdomen, flanks or thighs between September 1, 2013 and November 302013 was performed. Inclusion criteria: a) women between 25 and 45 years old; b) body mass index between 23 and 27 ; c) no systemic pathologies; d) no daily medication or chronic treatment; e) no specific contraindications for lipocryolysis (Eg.: Raynaud's disease, Haxthausen's disease, Essential Cryoglobulinemia or skin lesions in the area of application, including dermatitis, urticaria, ulcers, wounds, zoster, severe varicose veins); f) no other aesthetic medicine or weight reduction procedures within 30 days prior to the first session.

Thirty-two medical records fulfilled the inclusion criteria, although only 28 were included in the study. 4 medical records 
were discarded: 1 patient refuse consent to include his clinical record in this study, 1 patient did not satisfy inclusion criterion f), and 2 patients were lost to follow-up.

Lipocryolysis was performed with Lipocryo®, Clinipro S.L., Sant Cugat del Vallès, Spain, and the gel used was Transonic Gel 250 ml, Transonic, Spain.

\section{Results}

We have observed: erythema in $100 \%$ of cases, pain in $71.43 \%$, hematoma in $17.86 \%$, paresthesias $3.57 \%$ and blisters in $3.57 \%$. Figure 1 shows the absolute frequencies of the witnessed adverse effects.

\section{Discussion}

There is very little evidence regarding the adverse effects of lipocryolysis. In addition, the few studies which have explored them analyzed a very small sample. Compared to the great number of subjects who have undergone lipocryolysis treatments around the world in the past 5 years, the total population on which adverse effects have been studied is tiny. The mild, reversible, and usual adverse effects of lipocryolysis are known. However, are these a good approximation to real post-treatment complications, or is it that the total sample on which adverse effects have been assessed was too small?

Thousands of lipocryolysis treatments are performed around the world every day, and even if only empirically, no evidence of greater or different complications comes up. However, new adverse effects are reported occasionally, and although they are extremely rare, they should not be overlooked. A good example of this are the two cases of paradoxical adipose hyperplasia (a very rare condition with no identified risk factors) recently reported by Jailan et al [13].

New adverse effects studies with specific designs should be carried out in order to provide solid data. Based on the current status of the knowledge of the adverse effects of lipocryolysis, we believe that prospective, observational and simple studies enabling the assessment of a great number of subjects with very good follow-up capabilities are needed.

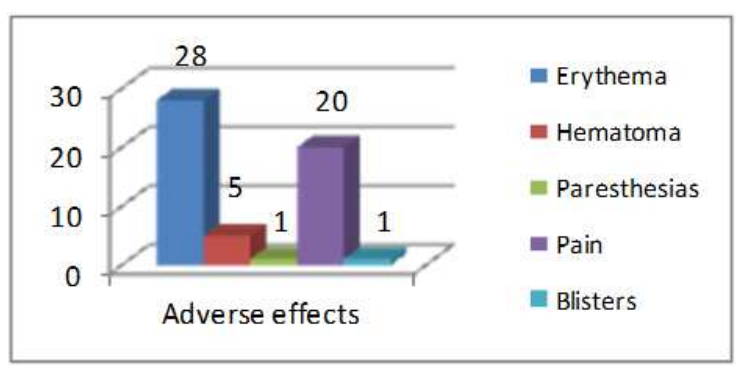

Figure 1. Adverse effects: absolute frequencies.

\section{References}

[1] Stewart KJ, Stewart DA, Coghlan B, Harrison DH, Jones BM, Waterhouse N. Complications of 278 consecutive abdominoplasties. J Plast Reconstr Aesthet Surg 2006;59(11):1152-5.

[2] Anderson RR, Farinelli W, Laubach H, Manstein D, Yaroslavsky AN, Gubeli J III, et al. Selective photothermolysis of lipid-rich tissues: A free electron laser study Lasers Surg Med 2006;38(10):913-9.

[3] Narins RS, Tope WD, Pope K, Ross EV. Over treatment effects associated with a radio frequency tissue-tightening device: Rare, preventable, and correctable with subcision and autologous fat transfer. Dermatol Surg 2006;32(1):115-24.

[4] Teitelbaum SA, Burns JL, Kubota J, Matsuda H, Otto MJ, Shirakabe Y, et al. Noninvasive body contouring by focused ultrasound: safety and efficacy of the contour I device in a multicenter, controlled, clinical study Plast Reconstr Surg 2007;120(3):779-89; discussion 790.

[5] Laubach H, Watannabe K, Farinelli W, ZurakowskiD, Anderson RR. Selective Cryolysis: A Novel Method of Non-Invasive Fat Reduction Las in Surg and Med 2008;40:595-604.

[6] Avram MM, Harry RS. Cryolipolysis for subcutaneous fat layer reduction. Lasers Surg Med. 2009Dec;41(10):703-8. Erratum in: Lasers Surg Med 2012Jul;44(5):436.

[7] Pinto H, Arredondo E, Ricart- Jané D. Evaluation of adipocytic changes after a simil-lipocryolysis stimulus CryoLetters 2013;34(1):100-5.

[8] Zelickson B, Egbert B, Preciado J, Allison A, Springer K, Manstein D. Cryolipolysis ${ }^{\mathrm{TM}}$ for Noninvasive Fat Cell Destruction: Initial Results from a Pig Model. Dermatol Surg 2009;35(10):1462-70.

[9] Coleman SR, Sachdeva K, Egbert BM, Preciado J, Allison J. Clinical efficacy of noninvasive cryolipolysis and its effects on peripheral nerves. Aesthetic Plast Surg. 2009Jul;33(4):482-8.

[10] Riopelle J, Tsai MY, Kovack B. Lipid and Liver Function Effects of the Cryolipolysis Procedure in a Study of Male Love Handle Reduction Laser Surg Med 2009;S21:82.

[11] Klein KB, Zelickson B, Riopelle JG, Okamoto E, Bachelor EP, Harry RS, et al. Non-invasive cryolipolysis for subcutaneous fat reduction does not affect serum lipid levels or liver function tests SurgMed 2009Dec;41(10):785-90.

[12] Dierickx CC, Mazer JM, Sand M, Koenig S, Arigon V. Safety, tolerance, and patient satisfaction with noninvasive cryolipolysis DermatolSurg 2013Aug;39(8):1209-16.

[13] Jalian HR, Avram MM, Garibyan L, Mihm MC, Anderson RR. Paradoxical Adipose Hyperplasia After Cryolipolysis JAMA Dermatol 2014Jan;Doi 10.1001/Jama dermatol.2013.8071 [Epubahead of print]. 